\title{
Analysis on the impact of granularity of abrasive jet to flow field structure Xiao-feng YANG $^{\mathrm{a},{ }^{*}, \text { Lu WANG }}{ }^{\mathrm{b}}$
}

Suzhou Institute of Wuhan University, Suzhou, 215123 China

axfyang@whu.edu.cn, bfloweryes@sina.com

${ }^{*}$ Corresponding author

Keywords: abrasive jet, granularity, flow field structure, momentum, effect

Abstract. The granularity of abrasive jet is closely related to the jet force. The paper uses the FLUENT software simulation technology to analyze the flow field structure under the condition of jet with different abrasive granularity. It is found that single particle momentum increases along with the growth of granularity, so does the amplification. But this trend would stop when the granularity reaches certain value. Momentum reflects the strike capability, so, there is an upper limit correspondingly, inferring the abrasive granularity contributing to abrasive jet force. For certain conditions of abrasive jet, optimal granularity range exists, which brings an optimal jet force. The optimal granularity shall be related to the nozzle structure, jet pressure and particle material properties. The research provides an effective reference for the mastery of the motion law of abrasive jet and optimizing the abrasive jet technology.

\section{Introduction}

Water jet technology is widely applied in the field of cold cutting processing, whereas the abrasive jet, whose advantage is energy-efficient, has become the focus in the current of water jet technology [1-2].The technology is different from the processing technology such as flame cutting, electron beam, laser beam and plasma, it is a cold working methods with high energy beam. It not only possesses the advantages of pure water jet cutting such as environmental protection, cheap, but also compensates for the limitations of thermal cutting, who cannot be applied in some fields. As a high efficient, high qualified, safe, environmental emerging technology. It is a revolution in the field of cutting with a broad application prospect in a way [3-4].

In general, the cutting ability of abrasive jet is related to the factors such as the pump pressure, abrasive properties and particle size [5-7]. But the concrete motion law and the mechanism of those influence factors towards abrasive jet is still unclear, especially the mechanism how the abrasive granularity affects the properties of jetting. The article researches how the particle size affects abrasive jet flow field structure, which has a positive significance for mastering flow field regularity and enhancing the working efficiency of the abrasive jet.

\section{the influence of the abrasive granularity on flow field structure}

Abrasive water jet involves the multiphase flow of water and abrasive, a multiphase flow model is needed to describe it. The research selected the Eulerian multiphase model. Multiphase flow model includes DPM model, VOF model, Mixture model and Eulerian model. DPM doesn't fit the research for its' ignorance of the effect that particles had on continuous fluid motion. VOF model is suitable for 
the layered or free surface flow, which requires tiny speed differences between each phase. Mixture and Eulerian model are both suitable for the flow of mix or separation phases, the former is used when separated phases have a broad distribution. Eulerian model is used in the article for the reasons above, which promises calculation for each phase separately with much flexibility [8].

During the process of specific analysis, simulation boundary conditions adopts speed entrance. Define the abrasive density of $2600 \mathrm{~kg} / \mathrm{m} 3$, volume fraction of 0.5 . Changeable abrasive particle size of $0.05 \mathrm{~mm}, 0.1 \mathrm{~mm}$ and $0.2 \mathrm{~mm}, 0.3 \mathrm{~mm}$ and $0.4 \mathrm{~mm}$, the iteration times as 1000 times. flow field structure gained after computing and analysis is as follows:

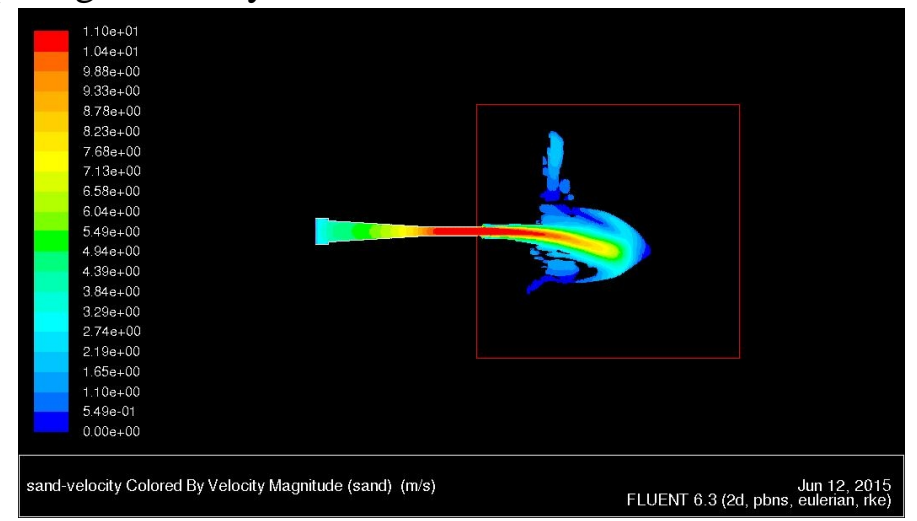

a. $\mathrm{d}=0.05 \mathrm{~mm}$

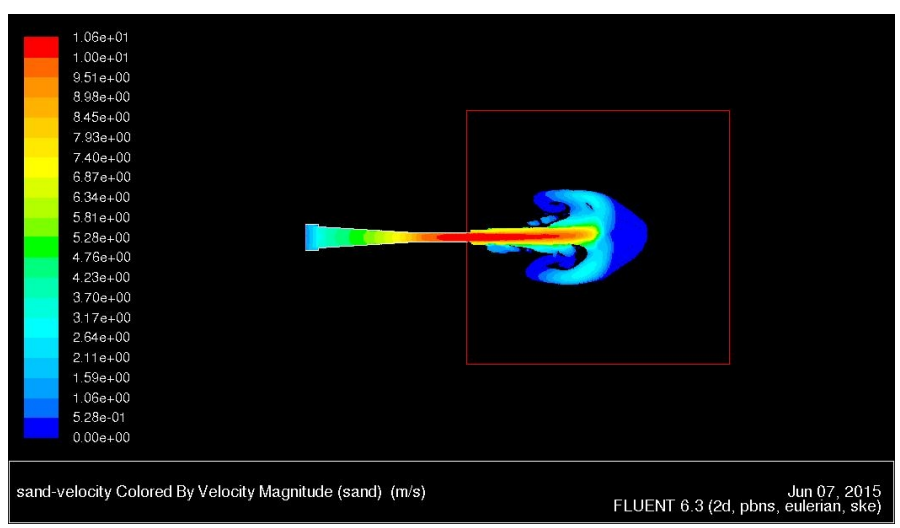

b. $\mathrm{d}=0.1 \mathrm{~mm}$

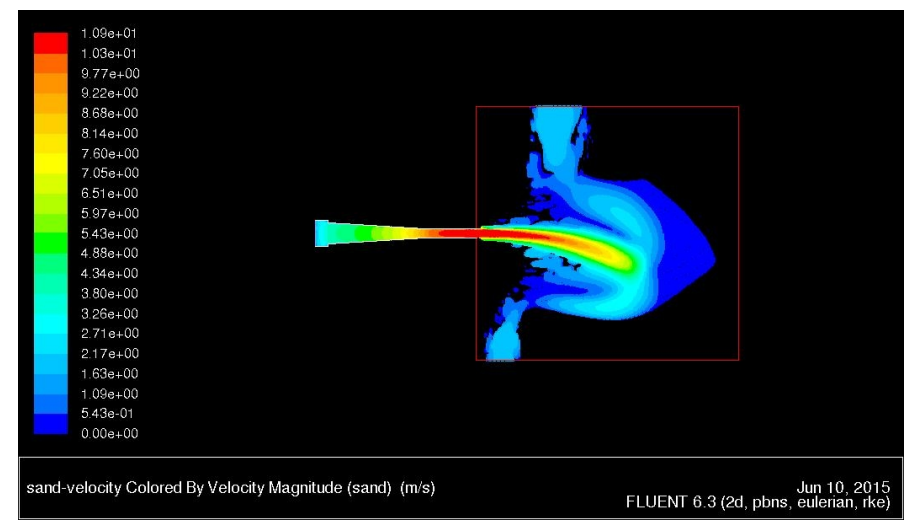

c. $\mathrm{d}=0.2 \mathrm{~mm}$ 

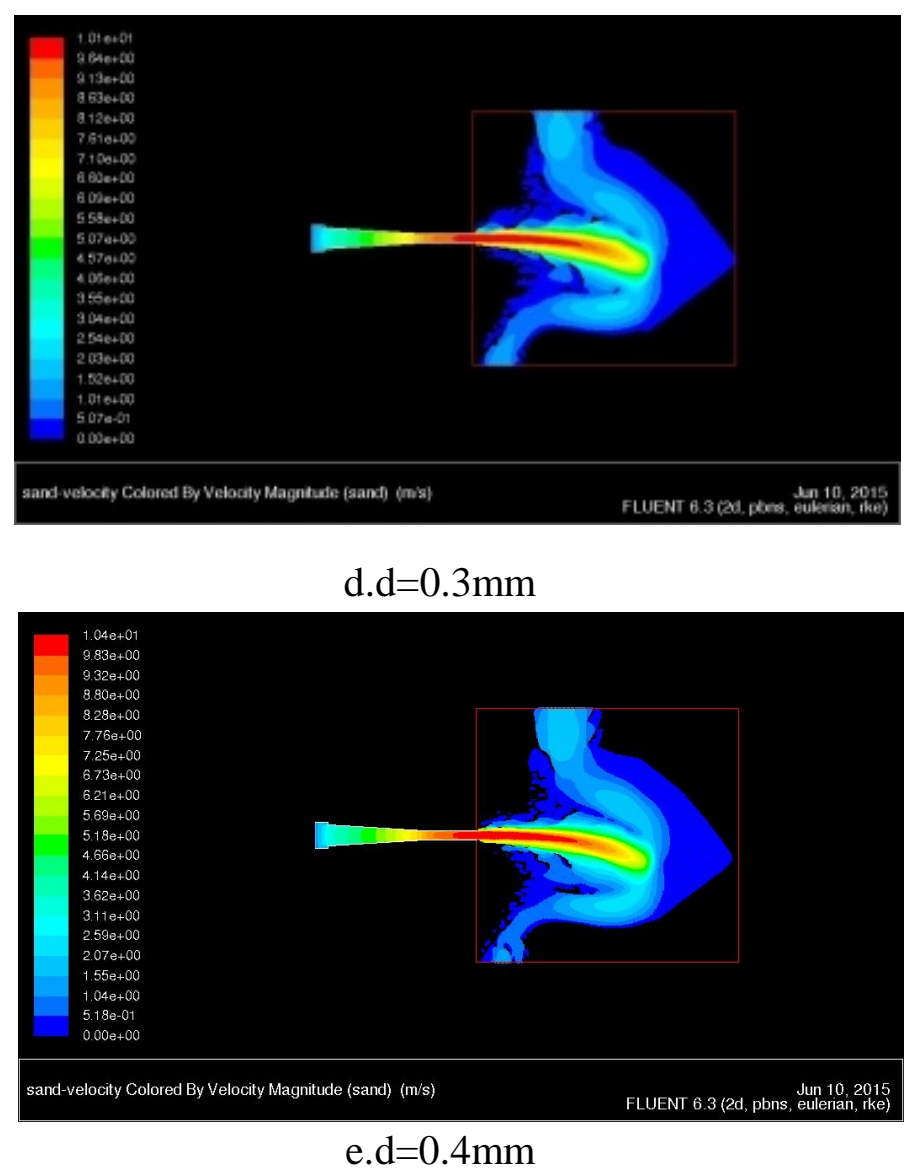

Fig. 1, the flow field structure under different abrasive particle diameter (d)

From the results of simulation, it is shown that the changes of the strength of the abrasive particles has little effect on the velocity of the abrasive core section under the same abrasive concentration. A slight decrease is observed along with the increase of the granularity.

The concentration is fixed, so that the calculation of momentum is counted by single particles' momentum. The calculation formula of particle momentum is:

$$
P=\frac{4}{3} \pi r^{3} V
$$

where $\mathrm{r}$ is the particle radius, $\mathrm{V}$ is the particle velocity.

The speed of the particles in the core section is calculated by FLUENT. So the five kinds of abrasive jet its relationship between the momentum and the granularity, are shown in the list below:

Table 1 momentum and granularity relational tables

\begin{tabular}{llllll}
\hline Granularity $(\mathrm{mm})$ & 0.05 & 0.1 & 0.2 & 0.3 & 0.4 \\
\hline Momentum $(\mathrm{kgm} / \mathrm{s})$ & 0.0572 & 0.0458 & 0.354 & 1.175 & 2.706 \\
\hline
\end{tabular}

It can be seen from the table above that in the condition when the granularity is less than 0.4 , the momentum of a single particle increases along with the granularity significantly, so does the amplification. But this trend would stop when the granularity reaches certain value. Momentum reflects 
the strike capability, so, there is an upper limit correspondingly, inferring the abrasive granularity contributing to abrasive jet force. According to the experimental experience, getting too rich at concentration would cause a block of abrasive particles inside the nozzle. For certain conditions of abrasive jet, optimal granularity range exists, which means an optimal jet force. The optimal granularity shall be related to the nozzle structure, jet pressure and particle material properties. It is required to combine theoretical analysis and experiment for the sake of ensuring the optimal granularity more accurately.

\section{conclusion}

The relation between the granularity of abrasive jet and jet force is of great significance to the improving the cutting ability of abrasive jet. The paper uses the FLUENT software simulation technology to analyze the flow field structure under the condition of jet with different abrasive granularity. It is found that single particle momentum increases along with the growth of granularity, so does the amplification. But this trend would stop when the granularity reaches certain value. Momentum reflects the strike capability, so, there is an upper limit correspondingly, inferring the abrasive granularity contributing to abrasive jet force. According to the experimental experience, getting too rich at concentration would cause a block of abrasive particles inside the nozzle. For certain conditions of abrasive jet, optimal granularity range exists, which brings an optimal jet force. The optimal granularity shall be related to the nozzle structure, jet pressure and particle material properties. The conclusion of the research has positive significance for improving the abrasive jet force, the jet technology and enhanceing the working efficiency of the abrasive jet.

\section{References}

[1] Wenying Xiang, Xiaohong Li, Yiyu Lu.2009. Experimental research on erosion of rock by submerged abrasive water jet. Journal of China University of Mining \& Technology(Social Science) (02): $240 \sim 243$.

[2] Zhonghou Shen.1998. Water jet theory and technology. University of petroleum press, Beijing.

[3] Gensheng Li, Zhongwei Huang, Shouzeng Tian.2011. Hydraulic jet fracturing theory and application. SciencePress, Beijing.

[4] Xiaojian Liu.2006. Research of abrasive slurry jet technology and mechanism. Shanghai University.

[5] Zhonghou Shen, Gensheng Li, Ruihe Wang.2002. The application and prospect of water jet technology in petroleum engineering. Chinese Engineering Science. 4(12): $60 \sim 65$.

[6] Shen Zhonghou, LI Gengsheng, Wang Zhiming. New Jet theory and prospects of application in petroleum engineering. Proceedings go the 13th World Petroleum Congress, Buenos Aires, Argentina, 1991, 397 $\sim 405$.

[7] Gensheng Li, Shen Zhonghou. High pressure water jet theory and its application research progress in petroleum engineering.oil exploration and development, 2005. 32(1): 96 99.

[8] Zhunong Liu. 2010. Numerical simulation and experimental research of high pressure water jet grinding solid materials. Xi'an University of Technology, Shanxi. 